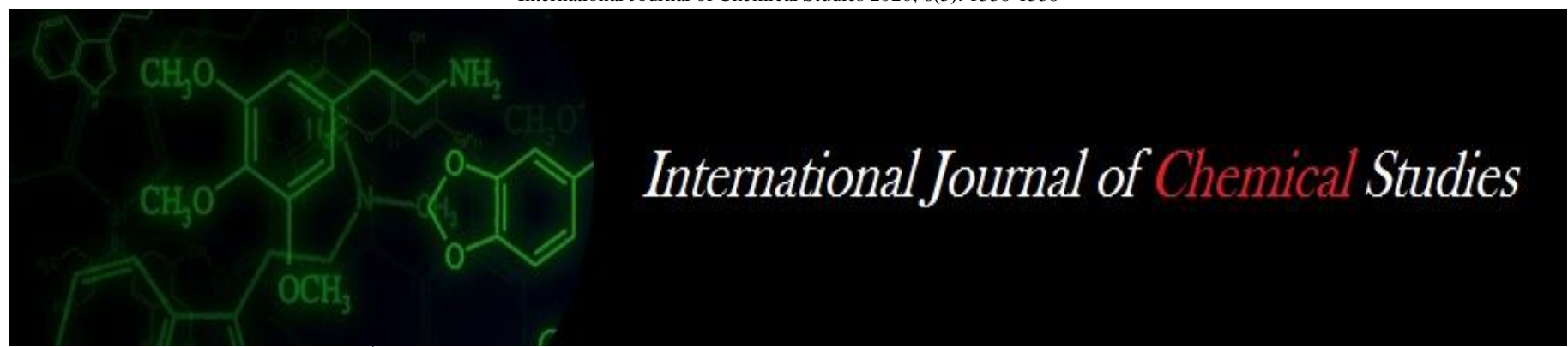

P-ISSN: 2349-8528

E-ISSN: 2321-4902

www.chemijournal.com

IJCS 2020; 8(5): 1336-1338

(C) 2020 IJCS

Received: 04-07-2020

Accepted: 08-08-2020

\section{Mallikarjun CJ}

College of Horticulture, UHS,

Bagalkot, Karnataka, India

Suvarna Patil

Assistant Professor of

Entomology, RHREC,

Dharwad, Karnataka, India

Athani SI

Professor of Fruit Science, Dean

Students Welfare, UHS

Bagalkot, Karnataka, India

Kotikal YK

Professor of Entomology and Director of Extension, UHS,

Bagalkot, Karnataka, India

Arunkumar B

Assistant Professor of

Entomology, RHREC,

Dharwad, Karnataka, India

Ramchandra NK

Professor of Post-Harvest

Technology and Head RHREC,

Dharwad, Karnataka, India

\section{Vinaykumar MM}

Assistant Professor of

Entomology, COH, Bagalkot,

Karnataka, India

Ambika DS

Assistant Professor, Plant

Pathology, COH, Bagalkot,

Karnataka, India
Corresponding Author:

Suvarna Patil

Assistant Professor of

Entomology, RHREC,

Dharwad, Karnataka, India

\title{
Efficacy of new insecticide molecules against mango leaf webber, Orthaga exvinacea Hampson (Pyralidae: Lepidoptera)
}

\author{
Mallikarjun CJ, Suvarna Patil, Athani SI, Kotikal YK, Arunkumar B, \\ Ramchandra NK, Vinaykumar MM and Ambika DS
}

DOI: https://doi.org/10.22271/chemi.2020.v8.i5s.10485

\begin{abstract}
The field experiment was conducted at Regional Horticultural Research and Extension Centre (RHREC), Kumbapur farm, Dharwad during 2018-19 to evaluate the efficacy of new molecules of insecticides against mango leaf webber in the established mango orchard. The cumulative mean of pest incidence fifteen days after second spray revealed that all the treatments showed comparative decrease in spread of the mango leaf webber infestation, but the treatment chlorantraniliprole $18.5 \%$ SC recorded least number of active webs per tree (2.17) and least number of larvae per web (2.82) and same was found effective in the management of $O$. exvinacea infesting mango over untreated control.
\end{abstract}

Keywords: mango, leaf webber, management, new insecticides

\section{Introduction}

The Mango (Mangifera indica L.), is described under dicotyledons which fall under the family Anacardiaceae and is classified under the order Sapindales. It is originated in the Indo-Burma region. It is considered as the "King of fruits". Mango flowers are borne on the inflorescence called flower panicles which appear to be creamy white to pinkish in colour. Now a days, incidence of leaf webber has been increasing in many areas. Mango leaf webber Orthaga exvinacea Hampson is considered to be a pest of occasional significance in the mango ecosystem. O. euadrusalis in the recent years are attaining a major pest status (Rajkumar et al., 2013) ${ }^{[4]}$. The extent of damage caused by this pest under favorable condition was estimated as 35 per cent (Srivastava and Tandon, 1980) ${ }^{[6]}$. The heavily infested trees appear to be burnt like from a distance wherein the leaves are dried, webbed and filled with excreta. The early instars (i.e., $1^{\text {st }}$ and $2^{\text {nd }}$ instars) scrape the chlorophyll content of the leaves and later stage instars start forming the webs by webbing 3-4 leaves together and larvae are very active in their movement inside the web where they will have tunnels made up of silken webs to escape, hide and pupate inside the webbings itself in a silken cocoon like case covered with its excreta outside. So, this severe infestation results in complete failure in flower initiation and finally the yield is affected (Anon, 2017) ${ }^{[1]}$.

\section{Materials and Method}

The field experiment was conducted at Regional Horticultural Research and Extension Centre (RHREC), Kumbapur farm, Dharwad during 2018-19 to evaluate the efficacy of new molecules of insecticides against mango leaf webber in the established mango orchard with Alphanso variety with spacing of $7.5 \times 7.5 \mathrm{~m}$. The experiment was laid out in simple Randomized Completely Block Design with spacing of $7.5 \times 7.5 \mathrm{~m}$. The total number of treatment were ten with three replications. Each tree was considered as one treatment.

Observations recorded: Infested trees with minimum of 10 to 15 webbings were selected and tagged. Observations were recorded a day prior to application of treatments as precount and recorded number of webs per tree and number of larvae per web. Post treatment counts were taken for the presence of larvae in the web at 3, 7 and 15 days after treatment. On the basis of number of active webs per tree and number of larvae per web, the data were analyzed to arrive at conclusion regarding efficacy of various insecticidal treatments. Similarly, second 
spray was imposed after one-month of first spray. The new insecticide molecules used under study are listed in Table 1. The fruit yield per tree was also recorded from each treatment separately and economics for each treatment was computed on the basis of fruit yield per hectare and market price.

Statistical analysis: Data on management of mango leaf webber was statistically analyzed (ANOVA) applying Randomized Block Design by using the software - WASP.

Table 1: Treatment details

\begin{tabular}{|c|c|c|}
\hline S. No. & Treatments & Dosage \\
\hline T1 & Azadirachtin 10,000 ppm & $1.0 \mathrm{ml} / \mathrm{l}$ \\
\hline T2 & Flonicamid 50\% WG & $2.0 \mathrm{~g} / \mathrm{l}$ \\
\hline T3 & Flubendiamide 20\% WG & $0.25 \mathrm{~g} / \mathrm{l}$ \\
\hline T4 & Lambda cyhalothrin 5\% EC & $0.5 \mathrm{ml} / 1$ \\
\hline T5 & Profenophos 50\% EC & $1.0 \mathrm{ml} / 1$ \\
\hline T6 & Fipronil 5\% SC & $1.0 \mathrm{ml} / 1$ \\
\hline T7 & Chlorantraniliprole $18.5 \% \mathrm{SC}$ & $0.2 \mathrm{ml} / 1$ \\
\hline T8 & Cyantraniliprole $10.26 \%$ OD & $1.2 \mathrm{ml} / 1$ \\
\hline T9 & Quinalphos 25\% EC (Standard check) & $2.0 \mathrm{ml} / 1$ \\
\hline T10 & Untreated Control & - \\
\hline
\end{tabular}

\section{Results and Discussion}

Observations were recorded on one day before imposing the treatment and 3, 7, 15 days after application of first and second spray.

Number of active webs per tree: The treatments chlorantraniliprole $18.5 \%$ SC and cyantraniliprole $10.26 \%$ OD observed to be the best, as least number of active webs per tree were 5.33 and 5.46 respectively. However, the treatments flubendiamide $20 \%$ WG was on par with lambda cyhalothrin 5\% EC and profenophos 50\% EC were next best. (Table 2). Shivmurthy (2014) [5] reported that chlorantraniliprole 0.03 per cent recorded the least number of active webs and was significantly different (4.34 active webs tree $^{-1}$ ) from all other treatments. Flubendiamide 0.01 per cent and lambdacyhalothrin 0.005 per cent (5.34 and 6.34 active webs tree ${ }^{-1}$ respectively) were the next best treatments and were significantly different from all other treatments.

Number of larvae per web: The treatments of chlorantraniliprole $18.5 \% \mathrm{SC}$ and cyantraniliprole $10.26 \%$ OD were recorded least number of larvae per web 2.50 and 2.88 respectively. However, the treatments flubendiamide $20 \%$ WG which was on par with lambda cyhalothrin $5 \%$ EC and profenophos $50 \%$ EC found next best. On the other hand, the treatments azadirachtin $10,000 \mathrm{ppm}$ and quinalphos $25 \%$ EC, the treatment flonicamid 50\% WG and fipronil 50\% EC observed to be the least effective and untreated control treatment recorded increase in number of larvae per web. Similar studies were reported by Shivamurthy (2014) ${ }^{[5]}$ that the lowest larval population was recorded in chlorantraniliprole 0.03 per cent, flubendiamide 0.01 per cent and azadirachtin one per cent $(4.00,4.33$ and 4.67 larvae web${ }^{1}$, respectively) in treated trees (Table 3 ).

Good yield of cabbage heads was recorded in chlorantraniliprole 18.50 SC treated plots against S. litura (Prathiban et al., 2014) ${ }^{[3]}$. These reports are in confirmity with the present findings in which chlorantraniliprole 0.002 per cent showed its superior efficacy to manage the shoot webber than other treatments. Chlorantraniliprole, cyantraniliprole and flubendiamide are new molecules and selective insecticides which are belonging to diamide group, their mode of action is by activating the insect ryanodine receptors (RyRs) which stimulates the release and depletion of intracellular calcium stores from the sacroplasmic reticulum of muscle cells, causing impaired muscles regulation, paralysis and ultimately death of sensitive species (Cordova et. al. 2006) ${ }^{[2]}$.

Cost economics and fruit yield data analysis indicated that among the different treatments, chlorantraniliprole $18.5 \%$ SC recorded highest fruit yield of $69 \mathrm{q} / \mathrm{ha}$ with highest B: C ratio (4.94) as compared to other treatments. Similarly, cyantraniliprole $10.26 \%$ OD recorded fruit yield of $68.20 \mathrm{q} / \mathrm{ha}$ with B: C ratio (4.37), lambda cyhalothrin 5\% EC and flubendiamide $20 \%$ WG were next best insecticides and quite promising for the management of leaf webber. But, flonicamid 50\% WG and fiprionil 5\% EC recorded least B:C ratio of 3.27 and 3.02 respectively, when compared to other newer insecticides (Table 4). Chlorantraniliprole is a new molecule and selective insecticide which are belonging to diamide group, their mode of action is by activating the insect ryanodine receptors (RyRs) which stimulates the release and depletion of intracellular calcium stores from the sacroplasmic reticulum of muscle cells, causing impaired muscles regulation, paralysis and ultimately death of sensitive species. It has very low toxicity for mammals (both acute and chronic), high intrinsic activity on target pests, strong ovilarvicidal and larvicidal properties, long lasting crop protection and no cross-resistance to any existing insecticide and also which are safer, target specific, less persistent, having green label and shows extremely strong insecticidal activity against Lepidopteran insect pests and is very safe to non-target organisms and effective even at low dosages. Based on the results of field experiment treatment chlorantraniliprole $18.5 \%$ SC @ 0.2 ml/l recorded least mean active number of webs per tree (2.82) and least mean number of larvae per web (2.17) found effective in the management of O. exvinacea infesting mango over control.

Table 2: Efficacy of new insecticides on number of active webs per tree in mango

\begin{tabular}{|c|c|c|c|c|c|c|c|c|c|}
\hline \multirow{3}{*}{ Treatments } & \multirow{3}{*}{ Dose } & \multicolumn{7}{|c|}{ Mean no. of active webs/tree } & \multirow{3}{*}{ Mean } \\
\hline & & & \multicolumn{3}{|c|}{ First spray } & \multicolumn{3}{|c|}{ Second spray } & \\
\hline & & DBS & 3 DAS & 7 DAS & 15 DAS & 3 DAS & 7 DAS & 15 DAS & \\
\hline T1 - Azadirachtin $10,000 \mathrm{ppm}$ & $1 \mathrm{ml} / 1$ & $15.15(3.89)$ & $12.97(3.60)^{\mathrm{d}}$ & $10.20(3.19)^{\mathrm{d}}$ & $8.02(2.83)^{\mathrm{c}}$ & $8.99(3.00)^{\mathrm{de}}$ & $7.62(2.76)^{\mathrm{c}}$ & $5.00(2.34)^{\mathrm{d}}$ & 8.8 \\
\hline $50 \% \mathrm{WG}$ & $2 \mathrm{~g} / \mathrm{l}$ & $14.36(3.80)$ & $14.09(3.75)^{\mathrm{e}}$ & $13.09(3.61)^{\mathrm{f}}$ & $11.69(3.41)^{\mathrm{e}}$ & $13.35(3.64)^{f}$ & $11.63(3.41)^{\mathrm{d}}$ & $8.28(2.96)^{\mathrm{e}}$ & 12.02 \\
\hline T3 - Flubendiamide $20 \% \mathrm{WG}$ & $0.25 \mathrm{~g} / 1$ & $15.15(3.89)$ & $11.84(3.44)^{\mathrm{bc}}$ & $7.99(2.82)^{\mathrm{b}}$ & $5.27(2.29)^{\mathrm{a}}$ & $6.76(2.59)^{\mathrm{bc}}$ & $3.74(1.93)^{\mathrm{a}}$ & $2.12(1.61)^{\mathrm{bc}}$ & 6.29 \\
\hline T4 - Lambda cyhalothrin 5\% EC & $0.5 \mathrm{ml} / 1$ & 16.23 & $12.88(3.58)^{\mathrm{cd}}$ & $9.47(3.07)^{\mathrm{c}}$ & $6.38(2.52)^{\mathrm{b}}$ & $7.66(2.76)^{\mathrm{cd}}$ & $4.88(2.21)^{\mathrm{b}}$ & $2.33(1.67)^{\mathrm{bc}}$ & 7.27 \\
\hline T5 - Profenophos 50\% EC & $1.0 \mathrm{ml} / 1$ & 14. & 13.54 & $10.16(3.18)^{\mathrm{d}}$ & $6.64(2.57)^{\mathrm{b}}$ & $7.89(2.79)^{\mathrm{cd}}$ & $4.92(2.22)^{\mathrm{b}}$ & $3.20(1.91)^{\mathrm{c}}$ & 7.73 \\
\hline $15 \% \mathrm{SC}$ & $1.0 \mathrm{ml} / \mathrm{l}$ & 14.40 & $14.18(3.76)^{\mathrm{e}}$ & $13.04(3.61)^{\mathrm{f}}$ & $12.08(3.47)^{\mathrm{e}}$ & $13.43(3.66)^{\mathrm{f}}$ & $11.53(3.40)^{\mathrm{d}}$ & $9.50(3.14)^{\mathrm{e}}$ & 12.29 \\
\hline T7 -Chlorantrani & $0.2 \mathrm{ml} / 1$ & $15.60(3.94)$ & $10.38(3.22)^{\mathrm{a}}$ & $7.58(2.75)^{\mathrm{ab}}$ & $5.19(2.27)^{\mathrm{a}}$ & $4.90(2.20)^{\mathrm{a}}$ & $2.29(1.51)^{\mathrm{a}}$ & $1.63(1.21)^{\mathrm{ab}}$ & 5.33 \\
\hline T8-Cyantranilip & $1.2 \mathrm{ml} / 1$ & $57(4.07)$ & $11.70(3.42)^{\mathrm{b}}$ & $7.23(2.68)^{\mathrm{a}}$ & $4.98(2.23)^{\mathrm{a}}$ & $5.89(2.41)^{\mathrm{ab}}$ & $2.03(1.43)^{\mathrm{a}}$ & $0.98(1.45)^{\mathrm{a}}$ & 5.46 \\
\hline T9-Quinalp & $2.0 \mathrm{ml} / 1$ & $14.25(3.78)$ & $13.00(3.60)^{\mathrm{d}}$ & $11.05(3.47)^{\mathrm{e}}$ & $8.58(3.25)^{\mathrm{d}}$ & $9.58(3.09)^{\mathrm{e}}$ & $7.84(2.80)^{\mathrm{c}}$ & $7.77(2.87)^{\mathrm{e}}$ & 9.68 \\
\hline T10 - Untre & - & $14.10(3.76)$ & $15.47(3.93)^{\mathrm{f}}$ & $18.12(4.25)^{\mathrm{g}}$ & $20.21(4.49)^{\mathrm{f}}$ & $22.36(4.73)^{\mathrm{g}}$ & $23.00(4.80)^{\mathrm{e}}$ & $23.55(4.90)$ & 20.45 \\
\hline & & \multirow{2}{*}{ NS } & \begin{tabular}{|l|}
0.04 \\
\end{tabular} & 0.03 & 0.03 & 0.7 & 0.06 & 0.07 & \\
\hline $\mathrm{CD}$ at $5 \%$ & & & 0.12 & 0.11 & 0.09 & 0.22 & 0.18 & 0.22 & \\
\hline
\end{tabular}


DBS - day before spray Figures in the parenthesis are square root of $(x+1)$ transformed values and DMRT $(p=0.05)$

DAS - day after spray S.Em \pm - Standard error mean, C.D. - Critical differenceat 5 per cent

Table 3: Efficacy of new insecticides on number of larvae per web in mango - First Spray

\begin{tabular}{|c|c|c|c|c|c|c|c|c|c|}
\hline \multirow{3}{*}{ Treatments } & \multirow{3}{*}{ Dose } & \multicolumn{7}{|c|}{ Mean no. of larvae/ web } & \multirow{3}{*}{ Mean } \\
\hline & & DBS & \multicolumn{3}{|c|}{ First spray } & \multicolumn{3}{|c|}{ Second spray } & \\
\hline & & DBS & 3 DAS & 7 DAS & 15 DAS & 3 DAS & 7 DAS & 15 DAS & \\
\hline T1 - Azadirachtin 10,000 ppm & $1 \mathrm{ml} / \mathrm{l}$ & $8.09(2.84)$ & $6.08(2.46)^{\mathrm{c}}$ & $4.87(2.20)^{\mathrm{cd}}$ & $3.26(1.80)^{\mathrm{e}}$ & $5.95(2.44)^{\mathrm{c}}$ & $4.80(2.19)^{\mathrm{c}}$ & $3.41(1.85)^{\mathrm{cd}}$ & 4.73 \\
\hline T2 - Flonicamid 50\% WG & $2 \mathrm{~g} / \mathrm{l}$ & $7.15(2.67)$ & $7.09(2.66)^{\mathrm{d}}$ & $5.57(2.36)^{\mathrm{f}}$ & $5.16(2.27)^{\mathrm{g}}$ & $9.68(3.11)^{\mathrm{e}}$ & $8.01(2.83)^{\mathrm{e}}$ & $6.41(2.53)^{\mathrm{e}}$ & 6.99 \\
\hline T3 - Flubendiamide $20 \%$ WG & $0.25 \mathrm{~g} / 1$ & $6.98(2.64)$ & $5.37(2.31)^{\mathrm{b}}$ & $3.82(2.61)^{\mathrm{b}}$ & $1.14(1.06)^{\mathrm{b}}$ & $4.95(2.22)^{\mathrm{ab}}$ & $3.56(1.89)^{\mathrm{b}}$ & $1.06(1.03)^{\mathrm{b}}$ & 3.31 \\
\hline T4 - Lambda cyhalothrin 5\% EC & $0.5 \mathrm{ml} / \mathrm{l}$ & $8.02(3.00)$ & $5.64(2.37)^{\mathrm{bc}}$ & $4.49(2.11)^{\mathrm{c}}$ & $2.32(1.50)^{\mathrm{c}}$ & $5.30(2.30)^{\mathrm{bc}}$ & $4.25(2.06)^{\mathrm{c}}$ & $2.80(1.67)^{\mathrm{c}}$ & 4.13 \\
\hline T5 - Profenophos 50\% EC & $1.0 \mathrm{ml} / \mathrm{l}$ & $7.00(2.65)$ & $5.60(2.36)^{\mathrm{bc}}$ & $5.13(2.26)^{\mathrm{de}}$ & $2.86(1.69)^{\mathrm{d}}$ & $5.85(2.42)^{\mathrm{c}}$ & $4.68(2.16)^{\mathrm{c}}$ & $3.26(1.81)^{\mathrm{c}}$ & 4.56 \\
\hline T6 - Fipronil 5\% SC & $1.0 \mathrm{ml} / \mathrm{l}$ & $9.08(2.84)$ & $7.27(2.69)^{\mathrm{d}}$ & $6.00(2.44)^{\mathrm{ef}}$ & $5.07(2.25)^{\mathrm{g}}$ & $10.08(3.17)^{\mathrm{e}}$ & $8.80(2.97)^{\mathrm{e}}$ & $6.54(2.56)^{\mathrm{e}}$ & 7.29 \\
\hline T7 -Chlorantraniliprole $18.5 \% \mathrm{SC}$ & $0.2 \mathrm{ml} / 1$ & $7.09(2.66)$ & $4.65(2.15)^{\mathrm{a}}$ & $2.25(1.5)^{\mathrm{ab}}$ & $1.00(1.00)^{\mathrm{ab}}$ & $4.29(2.07)^{\mathrm{a}}$ & $2.00(1.41)^{\mathrm{b}}$ & $0.78(0.88)^{\mathrm{b}}$ & 2.50 \\
\hline T8 -Cyantraniliprole $10.26 \%$ OD & $1.2 \mathrm{ml} / \mathrm{l}$ & $6.91(2.63)$ & $5.35(2.31)^{\mathrm{b}}$ & $3.42(1.84)^{\mathrm{a}}$ & $0.89(0.94)^{\mathrm{a}}$ & $4.75(2.18)^{\mathrm{ab}}$ & $2.58(1.70)^{\mathrm{a}}$ & $0.30(0.55)^{\mathrm{a}}$ & 2.88 \\
\hline T9-Quinalphos $25 \%$ EC & $2.0 \mathrm{ml} / \mathrm{l}$ & $7.11(2.67)$ & $6.24(2.49)^{\mathrm{c}}$ & $5.02(2.24)^{\mathrm{d}}$ & $4.21(2.05)^{\mathrm{f}}$ & $6.93(2.63)^{\mathrm{d}}$ & $5.73(2.39)^{\mathrm{d}}$ & $4.03(2.01)^{\mathrm{d}}$ & 5.36 \\
\hline T10 - Untreated Control & - & $6.89(2.62)$ & $8.05(2.83)^{\mathrm{e}}$ & $8.65(2.94)^{\mathrm{g}}$ & $10.79(3.28)^{\mathrm{h}}$ & $16.80(4.10)^{\mathrm{f}}$ & $18.00(4.24)^{\mathrm{f}}$ & $19.30(4.39)^{\mathrm{f}}$ & 14.75 \\
\hline S.Em \pm & & NS & 0.04 & 0.03 & 0.03 & 0.05 & 0.06 & 0.07 & \\
\hline CD at $5 \%$ & & & 0.13 & 0.09 & 0.09 & 0.16 & 0.18 & 0.21 & \\
\hline
\end{tabular}

DBS - day before spray Figures in the parenthesis are square root of $(x+1)$ transformed values and DMRT $(p=0.05)$

DAS - day after spray S.Em \pm - Standard error mean, C.D. - Critical differenceat 5 per cent

Table 4: Cost economics of new insecticides in management of mango leaf webber during 2018-19

\begin{tabular}{|c|c|c|c|c|c|c|c|}
\hline S. No. & Treatments & Dosage & Yield (q/ha) & $\begin{array}{c}\text { Total cost (Rs./ } \\
\text { ha) }\end{array}$ & $\begin{array}{c}\text { Gross returns (Rs./ } \\
\text { ha) }\end{array}$ & $\begin{array}{l}\text { Net returns } \\
\text { (Rs./ ha) }\end{array}$ & $\begin{array}{l}\mathrm{B}: \mathrm{C} \\
\text { ratio }\end{array}$ \\
\hline 1 & Azadirachtin $10,000 \mathrm{ppm}$ & $1.0 \mathrm{ml} / \mathrm{l}$ & 54.78 & $5,55,711$ & $2,25,920$ & $1,63,409$ & 3.93 \\
\hline 2 & Flonicamid 50\% WG & $2.0 \mathrm{~g} / 1$ & 49.50 & 64,900 & $1,98,000$ & $1,33,100$ & 3.27 \\
\hline 3 & Flubendiamide $20 \% \mathrm{WG}$ & $0.25 \mathrm{~g} / 1$ & 63.40 & 55,199 & $2,53,600$ & $1,98,433$ & 4.37 \\
\hline 4 & Lambda cyhalothrin 5\% EC & $0.5 \mathrm{ml} / \mathrm{l}$ & 60.12 & 55,167 & $2,40,480$ & $1,85,281$ & 4.36 \\
\hline 5 & Profenophos 50\% EC & $1.0 \mathrm{ml} / \mathrm{l}$ & 56.48 & 55,596 & $1,84,200$ & $1,69,908$ & 4.03 \\
\hline 6 & Fipronil 5\% SC & $1.0 \mathrm{ml} / \mathrm{l}$ & 45.48 & 55,596 & $1,81,920$ & $1,26,324$ & 3.05 \\
\hline 7 & Chlorantraniliprole $18.5 \%$ SC & $0.2 \mathrm{ml} / 1$ & 69.55 & 62,429 & $2,78,200$ & $2,21,880$ & 4.94 \\
\hline 8 & Cyantraniliprole $10.26 \%$ OD & $1.2 \mathrm{ml} / \mathrm{l}$ & 68.20 & 56,320 & $2,72,800$ & $2,10,371$ & 4.60 \\
\hline 9 & Quinalphos 25\% EC & $2.0 \mathrm{ml} / \mathrm{l}$ & 46.05 & 56,012 & $2,19,120$ & $1,28,604$ & 3.31 \\
\hline 10 & Untreated Control & - & 40.33 & 54,000 & $1,61,320$ & $1,07,320$ & 2.99 \\
\hline
\end{tabular}

B: C - Benefit cost ratio

\section{References}

1. Anonymous. Horticultural statistics, NHB, Gurugram, Haryana, 2017, 142p.

2. Cordova D, Benner EA, Sacher MD, Rauh JJ, Sopa JS, Lahm GP et al., Anthranilic diamides: A new class of insecticides with a novel mode of action, ryanodine receptor activation, Pestic. Biochem. Physiol. 2006; 84:196-214A.

3. Prathiban P, Baskaran RKM, Thangavel K. Bio-efficacy of emamectin benzoate $5 \mathrm{WG}$ against tobacco caterpillar and diamondback moth in cabbage. Pesticide Res. J. 2014; 26(2):175-180.

4. Rajkumar B, Gundapp Khan RM, Kumar HK. Integrated pest management for enhancing quality production of subtropical fruits under high density planting with canopy modification. In: canopy management and high density planting in subtropical fruit crops, 2013 Eds. Singh V. K and Ravishankar H, CISH, Lucknow, 2013, 269p.

5. Shivamurthy. Population dynamics and management of shoot webber and hoppers infesting mango using safer molecules, M. Sc. (Agri.) thesis, Kerala Agricultural University, Vellayani, and Thiruvananthapuram, 2014.

6. Srivastava RP, Tandon PL. Studies of insect pathogens on mango leaf webber, Orthaga euadrusalis Walker (Lepidoptera: Pyralidae). Entomon. 1980; 5:219-221. 